\title{
Mouse Cancer Genome Anatomy Project
}

National Cancer Institute

\section{Source}

National Cancer Institute. Mouse Cancer Genome Anatomy Project. NCI Thesaurus.

Code C15944.

Similar to the human CGAP. The overall goals of the m-CGAP are: a comparative assessment of mouse cancer stages with those of cognate human cancers, both to validate the mouse models and to promote crossfeeding in the identification of genes that characterize those cancers; the discovery of new genes and gene pathways involved in the stages of carcinogenesis. 